\title{
Análisis de género de la producción científica española sobre drogodependencias en biomedicina 1999-2004
}

\author{
Gregorio González-Alcaide; Juan Carlos Valderrama-Zurián; Carolina Navarro-Molina; \\ Adolfo Alonso-Arroyo; Máxima Bolaños-Pizarro; Rafael AleiXandre-Benavent
}

Instituto de Historia de la Ciencia y Documentación López Piñero. Universitat de València-CSIC. Valencia.

Enviar correspondencia a:

Juan Carlos Valderrama Zurián. Instituto de Historia de la Ciencia y Documentación López Piñero. Av. Blasco Ibáñez, 15. Valencia 46010.

e-mail: Juan.Valderrama@uv.es

Recibido: Septiembre 2006.

Aceptado: Noviembre 2006.

\section{RESUMEN}

Fundamento y objetivo: La preocupación por favorecer la igualdad de género constituye uno de los ámbitos prioritarios objeto de atención por parte de los gobiernos y los organismos gestores de las políticas científicas, destacándose la importancia de incluir la variable del sexo en los análisis evaluativos de la actividad científica y tecnológica. Se realiza un estudio comparativo desagregado por sexos de la producción científica de los investigadores españoles de elevada productividad en el área de las drogodependencias.

Material y método: Se ha identificado el sexo de los 338 autores que han publicado más de cuatro artículos distintos durante el período 1999-2004 indexados en las bases de datos IME/Índice Médico Español y SCI/Science Citation Index, analizando comparativamente su productividad y patrones de colaboración considerando la variable sexo.

Resultados: Entre los grandes productores (> 9 trabajos) del ámbito de las drogodependencias predominan los hombres $(70 \%)$ frente a las mujeres $(30 \%)$. Entre los productores moderados (5-9 trabajos), el 57\% de los autores identificados fueron hombres frente a un $43 \%$ de mujeres. Se han observado diferencias estadísticamente significativas entre hombres y mujeres en los grandes productores en cuanto al número de trabajos publicados y personas con las que han colaborado.

Conclusiones: En el área de las drogodependencias no existe una igualdad de género, especialmente cuando se considera la élite de la investigación. Resulta fundamental profundizar en estudios que evalúen de forma desagregada por sexos la productividad científica para acometer las medidas correctoras necesarias que permitan alcanzar la igualdad de género.

Palabras clave: Análisis de género, publicaciones científicas, drogodependencias, análisis bilbiométrico.

\section{ABSTRACT}

Background and objective: Concern for encouraging gender equality makes it one of the high priority spheres of action for governments and organisations responsible for instigating scientific policies, with particular importance being placed on including the gender variable in evaluative analyses of scientific and technological activity. A comparative study was made, broken down by gender, of the scientific output of Spanish researchers with a high production in the field of substance abuse.

Material and method: We identified the gender of 338 authors who had published more than four different articles during the period from 1999-2004 and which were indexed in the IME/Índice Médico Español and the SCI/Science Citation Index databases, making a comparative analysis of their output and collaboration patterns, based on the gender variable.

Results: In the area of substance abuse, of those with the highest output (> 9 papers), 70\% were men compared with $30 \%$ women. Among the average producers $(5-9$ papers), $57 \%$ of the authors identified were men and $43 \%$ women. Statistically significant differences were observed between men and women with the highest output with regard to the number of published works and those with whom they had collaborated.

Conclusions: There is no gender equality in the area of substance abuse, particularly when considering the top researchers. It is essential to make in-depth studies that evaluate scientific output, broken down by gender, in order to adopt the necessary corrective measures to eliminate the disparity between men and women.

Key words: Gender analysis, Scientific publications, Substance abuse, Bibliometric Analysis. 


\section{INTRODUCCIÓN}

A partir de la década de los 70 coincidiendo con el resurgir de los movimientos feministas en Estados Unidos aparece un decidido interés por conocer de forma precisa la participación de las mujeres en las actividades científicas ${ }^{1}$. En Europa fueron Alemania (1989), el Reino Unido (1993) y Dinamarca (1997) los primeros países en realizar informes estadísticos rigurosos para conocer el papel desempeñado por la mujer en estas actividades. Posteriormente, en 1998, la Dirección General de Investigación de la Unión Europea creó un grupo de trabajo sobre Mujeres y Ciencia, que elaboró el llamado informe ETAN, donde se analizaba la situación de las mujeres en este ámbito y se realizaban toda una serie de recomendaciones para promover la igualdad de género².

En España se han desarrollado algunos estudios puntuales centrados en la presencia de la mujer en las universidades ${ }^{3}$ y en el Consejo Superior de Investigación Científicas (CSIC) ${ }^{4}$, así como informes sobre la mujer en el sistema educativo español y en el ámbito científico tecnológico ${ }^{5}$. La preocupación por favorecer la igualdad de género a nivel gubernamental tiene como hito más destacado la creación de un organismo específico adscrito al Ministerio de Educación y Ciencia a través de la Secretaría General de Política Científica y Tecnológica, la Unidad de Mujeres y Ciencia, cuya misión es favorecer la incorporación de las mujeres en condiciones de igualdad al sistema científico y tecnológico español ${ }^{6}$.

Los informes y estudios realizados hasta la fecha han incidido fundamentalmente en datos estadísticos sobre educación y ocupación de puestos profesionales. Sin embargo, la evaluación de la igualdad de género en el desarrollo de las actividades científicas requiere la realización de estudios desagregados por sexo que cuantifiquen el desarrollo de esa actividad, lo que se puede realizar a través del análisis de las publicaciones científicas. El objetivo de este estudio es realizar un análisis bibliométrico considerando la variable sexo de los investigadores españoles que han publicado cinco o más trabajos en el campo de las drogodependencias, estudiando su producción científica y sus patrones de colaboración.

\section{MÉTODOS}

La información para la realización del estudio se ha obtenido seleccionado los trabajos recogidos en la base de datos IME/Índice Médico Español, y en la base de datos SCI/Science Citation Index. El proceso seguido ha sido el siguiente:

A) Identificación y selección de los artículos. Para la recuperación de las referencias pertinentes se ha aplicado un perfil de búsqueda y una metodología similar a la empleada en otros trabajos previos ${ }^{7}$, que ha permitido obtener 2.659 trabajos distintos sobre drogodependencias publicados a lo largo del sexenio 1999-2004, considerando el ámbito de las drogodependencias desde un punto amplio y que incluye estudios sobre receptores y fisiopatología.

B) Confección de una base de datos relacional. Con la información extraída se ha elaborado una base de datos relacional utilizando el software Microsoft Access, a fin de facilitar el análisis de la información bibliográfica, añadiendo un campo específico para indicar el sexo de los autores. Se han estudiado, siguiendo la clasificación de Crane ${ }^{8}$, los "grandes productores", aquellos que han publicado más de nueve trabajos y los "productores moderados", aquellos con un número de trabajos comprendido entre cinco y nueve. Para conocer el sexo de los autores se han consultado los directorios de personal de las organizaciones a los que están adscritos (conocidas a través de las firmas institucionales de los trabajos) o bien se ha accedido al texto completo de los artículos, donde es frecuente que se desarrolle el nombre completo de los autores.

C) Construcción de la red de coautorías, cálculo de indicadores y análisis estadístico desagregado por sexos. Con la información obtenida se ha realizado un análisis de redes sociales, construyendo la red de coautorías de los autores seleccionados para la realización del estudio. Cuando se hace referencia a un subgrupo de investigación dentro de la red se alude a los investigadores que mantienen una alta densidad de relaciones entre sí y un escaso número de conexiones con el exterior.

Los indicadores y medidas calculados para cada uno de los autores con el objeto de determinar la existencia de diferencias según el sexo han sido: productividad ( $n^{\circ}$ de trabajos), grado $\left(n^{\circ}\right.$ de autores distintos con los que se ha establecido colaboración, diferenciando si se trata de hombres o mujeres), intermediación (medida que determina si un autor aparece en el camino más corto que conecta a otros autores entre sí) y cercanía (evalúa la distancia existente de un autor con el resto de autores con los que está conectado). Así por ejemplo, según se muestra en la figura 1 , que recoge los vínculos de coautoría establecidos entre los grandes productores, Marta Torrens es una investigadora que cumple un destacado papel de "puente", ya que a través de ella se establece la conexión entre diferentes núcleos de investigadores que de otra forma quedarían aislados y Marta Rodríguez y María A. Aguilar son dos autoras "cercanas" entre sí, en tanto que han establecido un vínculo directo de coautoría, mientras que 


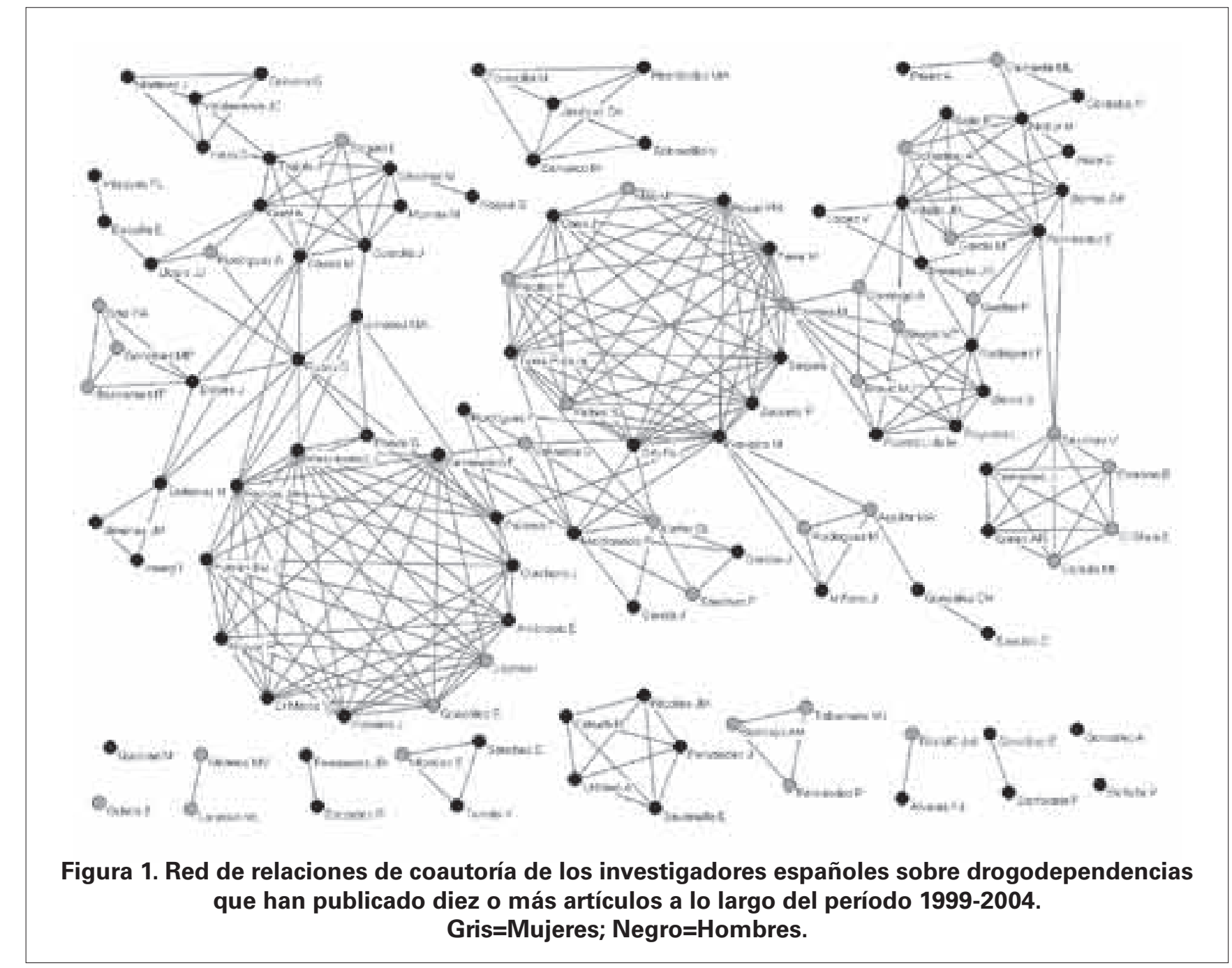

María Isabel Colado y Lidia Segura son autoras "lejanas", ya que están interconectadas, pero a través de un elevado número de autores intermedios. Se ha analizado asimismo la existencia de diferencias en relación con la adscripción institucional y geográfica por comunidades autónomas de los autores.

Para la construcción de la red de coautorías y el cálculo de la medidas de análisis de redes se ha utilizado el programa Pajek. El análisis estadístico se ha realizado con el paquete estadístico SPSS (versión 13.0), obteniéndose los valores descriptivos (media y desviación estándar) y utilizándose la t de student para la comparación de medias según sexo.

\section{RESULTADOS}

Se han identificado 338 investigadores que han firmado más de cuatro trabajos sobre drogodependencias, de los cuales 209 son hombres (62\%) y 129 mujeres (38\%). Considerando únicamente los "grandes productores" ( $n=117)$, el $70 \%$ son hombres ( $n=82)$ frente a un $30 \%$ de mujeres $(n=35)$. Entre los investigadores de "productividad moderada" $(n=221)$ el $57 \%$ son hombres $(n=127)$ frente a un $43 \%$ de mujeres $(n=94)$. La Comunidad de Madrid y Cataluña son las que reúnen un mayor número de mujeres investigadoras ( $n=35$ y $n=33$ respectivamente). En nueve Comunidades Autónomas no existe ninguna mujer "gran productora" (Andalucía, Canarias, Cantabria, Comunidad Foral de Navarra, País Vasco, Castilla-La Mancha, Extremadura, Baleares y La Rioja), no contando tampoco las cuatro últimas con "productoras moderadas". La Universidad Complutense de Madrid se sitúa en primer lugar en cuanto a número de investigadoras, existiendo en esta institución una igualdad entre el número de hombres $(n=18)$ y de mujeres $(n=18)$. En segundo lugar se sitúa el Institut Municipal d'Investigació Mèdica de Barcelona, que cuenta con una mayor número de grandes o moderadas productoras $(n=11)$ que de grandes o moderados productores $(n=7)$. A continuación destacan el Consejo Superior de Investigaciones Científicas, el Hospital Clínic i Provin- 
cial de Barcelona, la Universidad del País Vasco, la Universidad de Santiago de Compostela y la Universitat de València, con cinco mujeres en cada una de ellas.

El análisis estadístico efectuado que se recoge en la tabla 1 pone de manifiesto que entre los autores que han publicado diez o más trabajos existen diferencias estadísticamente significativas entre hombres y mujeres respecto al $n^{\circ}$ de trabajos publicados, al $n^{\circ}$ de personas con las que han colaborado y al $n^{\circ}$ de colaboraciones, diferencias que no se observan cuando se analizan estas medidas considerando únicamente los "productores moderados". Asimismo se han detectado diferencias en cuanto al sexo de los colaboradores, ya que los hombres colaboran más con otros hombres que las mujeres, que colaboran indistintamente tanto con hombres como con mujeres. Así, la media del número de colaboradores de los hombres con otros hombres es de 4,65 frente a una media de 3,81 de colaboración de las mujeres con hombres ( $t=2,237$; $g l=303,160 ; p=0,026)$, diferencias que aún son más acusadas entre los "grandes productores" $(6,79$ y 5,03 respectivamente). No existen diferencias en cuanto al número de mujeres que publican en revistas españolas (de difusión nacional e internacional) y extranjeras. En la figura 1 se representan de forma diferenciada por sexos las relaciones de coautoría establecidas entre los "grandes productores", pudiendo apreciarse los principales núcleos de investigadores, las posiciones que ocupan las mujeres en los mismos y los vínculos que han establecido. En este sentido, a pesar de que dentro de los subgrupos de grandes productores que se han identificado las mujeres son cuantitativamente una minoría, con sólo dos (Isabel Lastres y Sara González) y cuatro mujeres (Marta Mas, Roberta Pacifici, Simona Pichini y Marta Torrens) en los subgrupos de mayor tamaño, respectivamente, cabe resaltar la existencia de algunas destacadas investigadoras que ejercen un importante papel de intermediarias permitiendo la interconexión de diferentes subgrupos: Olga Valverde, Verónica Sánchez, Antonia Domingo, María Teresa Brugal, Brigitte L. Kieffer, María José Bravo y María A. Aguilar, además de la citada Marta Torrens. Destaca asimismo un subgrupo en el que cuatro de sus seis integrantes son mujeres (Esther O'Shea, Blanca Esteban, María Isabel Colado y Verónica Sánchez).

\section{DISCUSIÓN}

Con el presente estudio se ha pretendido realizar una aproximación a la distribución por sexos de los investigadores españoles del campo de las drogodependencias en biomedicina mediante el empleo de técnicas bibliométricas centradas en el análisis de la productividad y de indicadores de colaboración procedentes del análisis de redes sociales.

Algunas de las conclusiones más relevantes del estudio realizado son las siguientes:

Tabla 1. Análisis estadístico de la productividad y los patrones de colaboración de los investigadores españoles sobre drogodependencias 1999-2004.

\begin{tabular}{|c|c|c|c|c|c|c|c|}
\hline \multirow{2}{*}{$\begin{array}{l}\text { Grandes productores } \\
(\mathrm{n}=117)\end{array}$} & \multicolumn{2}{|c|}{ Media } & \multicolumn{2}{|c|}{ Desviación estándar } & \multirow{2}{*}{$\mathbf{t}$} & \multirow{2}{*}{ gl } & \multirow{2}{*}{$\mathbf{p}$} \\
\hline & $H(n=82)$ & $M(n=35)$ & H & M & & & \\
\hline $\mathrm{N}^{\circ}$ trabajos & 17,17 & 13,91 & 8,15 & 3,93 & 2,91 & 113,23 & 0,00 \\
\hline $\mathrm{N}^{\circ}$ colaboradores & 10,65 & 7,97 & 6,79 & 5,12 & 2,09 & 115 & 0,04 \\
\hline $\mathrm{N}^{\circ}$ de colaboraciones & 52,30 & 37,40 & 42,02 & 20,94 & 2,55 & 112,19 & 0,01 \\
\hline Intermediación & 0,01 & 0,01 & 0,02 & 0,02 & 0,96 & 115 & 0,34 \\
\hline Cercanía & 0,17 & 0,15 & 0,06 & 0,07 & 1,28 & 115 & 0,20 \\
\hline \multirow{2}{*}{$\begin{array}{l}\text { Productores moderados } \\
(\mathrm{n}=221)\end{array}$} & \multicolumn{2}{|c|}{ Media } & \multicolumn{2}{|c|}{ Desviación estándar } & \multirow{2}{*}{$\mathbf{t}$} & \multirow{2}{*}{ gl } & \multirow{2}{*}{$\mathbf{p}$} \\
\hline & $H(n=127)$ & $M(n=94)$ & H & M & & & \\
\hline$N^{0}$ trabajos & 6,20 & 6,16 & 1,29 & 1,31 & 0,21 & 219 & 0,83 \\
\hline $\mathrm{N}^{\circ}$ colaboradores & 4,68 & 5,26 & 3,31 & 3,93 & $-1,18$ & 219 & 0,24 \\
\hline $\mathrm{N}^{\circ}$ de colaboraciones & 14,07 & 16,84 & 11,26 & 13,09 & $-1,67$ & 219 & 0,09 \\
\hline Intermediación & 0,00 & 0,00 & 0,00 & 0,01 & 0,09 & 219 & 0,92 \\
\hline Cercanía & 0,12 & 0,13 & 0,07 & 0,07 & $-0,69$ & 219 & 0,49 \\
\hline
\end{tabular}


a) no existe una igualdad entre hombres y mujeres en cuanto a número de investigadores y trabajos publicados, diferencias que se acentúan en relación con la mayor productividad de los autores y que son especialmente significativas a nivel geográfico, con la inexistencia de mujeres grandes productoras en numerosas Comunidades Autónomas; b) A nivel institucional destaca la existencia de una paridad de sexo o el predominio de las mujeres en algunas instituciones, como la Universidad Complutense de Madrid y el Institut Municipal d'Investigació Mèdica de Barcelona; c) A pesar de que las mujeres "grandes productoras" son cuantitativamente menores a los hombres, el análisis de redes revela que son numerosas las mujeres que ocupan destacadas posiciones en la red de investigadores de elevada productividad, desempeñando por tanto, un importante papel de canalizadoras y difusoras de información, además de relacionarse indistintamente tanto con hombres como con mujeres.

La existencia de una paridad de mujeres y hombres en la realización de estudios universitarios y en la obtención del grado de doctor no implica ${ }^{5}$, según se ha observado, la existencia de una igualdad en lo relativo al número de investigadores que están generando una producción científica activa en forma de publicaciones. El porcentaje de mujeres investigadoras identificadas en el ámbito de las drogodependencias (38\%) sigue siendo sensiblemente inferior al de los hombres pese a que está por encima de otras áreas y de la media del número de mujeres investigadoras del área de la Biología y la Biomedicina del CSIC, que se situaba en 2001 en un 31,5\% y en 2003 en un $30,31 \%{ }^{4}$. Estos hechos justificarían decisiones como la del Ministerio de Educación y Ciencia de crear la Unidad de Mujeres y Ciencia ${ }^{6}$ y las políticas de promoción de la mujer en la convocatoria de proyectos competitivos, favoreciendo aquellos en los que el cociente de género mejora la media de su Programa Nacional, área o subprograma ${ }^{9}$. Estas actuaciones deberían corregir lo que algunos autores han denominado efecto "tijera" 5 por el que los hombres se ven favorecidos en la ocupación de los puestos superiores del escalafón científico y consiguientemente en el proceso de publicación ${ }^{10}$.

Cabe apuntar la existencia de diversas limitaciones en relación con los resultados y el estudio realizado: a) se han recogido únicamente los autores de elevada productividad (> 4 trabajos) sobre drogodependencias en biomedicina. Una visión completa de la investigación debería tener en cuenta todos los autores firmantes con independencia del área y de su número de trabajos. b) los artículos publicados en publicaciones periódicas sólo representan una parte de la investigación científica y un análisis exhaustivo exige analizar otros documentos como tesis doctorales, informes científico-técnicos o patentes. c) Es fundamental profundizar en estudios que evalúen de forma desagregada la participación de ambos sexos en las publicaciones científicas, así como aquellos que analicen su evolución a lo largo del tiempo, ya que aportan una información fundamental para conocer y acometer las medidas correctoras necesarias para evitar los desequilibrios de género, además de incidir en otros aspectos como el impacto de los trabajos publicados, el orden de las firmas y la existencia de diferencias en función de área de trabajo o de la categoría profesional.

\section{AGRADECIMIENTOS}

El presente estudio se ha llevado a cabo gracias al apoyo de las siguientes instituciones:

Plan Municipal de Drogodependencias. Concejalía de Sanidad. Ayuntamiento de Valencia.

Dirección General de Drogodependencias. Agencia Valenciana de Salud. Conselleria de Sanitat. Generalitat Valenciana.

Delegación del Gobierno para el Plan Nacional sobre Drogas. Ministerio de Sanidad y Consumo.

Consejo Superior de Investigaciones Científicas. Programa I3P cofinanciado por el Fondo Social Europeo.

\section{REFERENCIAS}

1. Cobo Bedia R. Género. En: Amorós C, editor. 10 palabras clave sobre mujer. Navarra: Verbo Divino; 2000. p. 5583.

2. ETAN Expert Working Group. Science Policies in the European Union: promoting excellence though mainstreaming gender equality. Bruselas: European Commission; 2000.

3. García de León MA. Las profesoras universitarias: el caso de una élite discriminada. Rev Complut Educ 1990; 1 : 355-72.

4. Consejo Superior de Investigaciones Científicas. Mujeres investigadoras del CSIC (2001, 2003, 2005). Disponible en: http://www.csic.es/mujer_ciencia.doc [Consultado 01-08-2006].

5. Mujer y Ciencia: la situación de las mujeres investigadoras en el sistema español de ciencia y tecnología. Madrid: Fundación Española para la Ciencia y la Tecnología; 2005.

6. Boletín Oficial del Estado. ORDEN de 7 de marzo, por la que se da publicidad al Acuerdo de Consejo de Ministros por el que se adoptan medidas para favorecer la igualdad entre mujeres y hombres. BOE $n^{\circ} 57.8$ de marzo de 2005, p. 8111-14.

7. González Alcaide G, Valderrama Zurián JC, Aleixandre Benavent R, Alonso Arroyo A, De Granda Orive JI, Villanueva Serrano S. Redes de coautoría y colaboración de las instituciones españolas en la producción científica 
sobre drogodependencias en biomedicina 1999-2004. Trast Adict 2006; 8: 78-114.

8. Crane D. Social structure in a group of scientists: a test of the "invisible college" hypothesis. Am Sociol Rev 1969; 34: 335-52.

9. Boletín Oficial del Estado. Resolución de 30 de noviembre de 2005, de la Secretaría de Estado de Universidades e Investigación, por la que se hace pública la convocato- ria de ayudas para la realización de proyectos de investigación, en el marco de algunos Programas Nacionales del Plan Nacional de Investigación Científica, Desarrollo e Innovación Tecnológica 2004-2007. BOE n² 294. 9 de diciembre de 2005, p. 40483-512.

10. Mauleón E, Bordons M. Productivity, impact and publication habits by gender in the area of Materials Science. Scientometrics 2006; 66: 199-218. 